\title{
Molecular differential diagnosis of follicular thyroid carcinoma and adenoma based on gene expression profiling by using formalin-fixed paraffin-embedded tissues
}

Aleksandra Pfeifer ${ }^{1,5+}$, Bartosz Wojtas ${ }^{1 \dagger}$, Malgorzata Oczko-Wojciechowska', Aleksandra Kukulska', Agnieszka Czarniecka ${ }^{2}$, Markus Eszlinger ${ }^{6}$, Thomas Musholt ${ }^{7}$, Tomasz Stokowy ${ }^{1,5}$, Michal Swierniak ${ }^{1,8}$, Ewa Stobiecka ${ }^{4}$, Dagmara Rusinek ${ }^{1}$, Tomasz Tyszkiewicz ${ }^{1}$, Monika Kowal ${ }^{1}$, Michal Jarzab ${ }^{3}$, Steffen Hauptmann ${ }^{9}$, Dariusz Lange ${ }^{4}$, Ralf Paschke ${ }^{6}$ and Barbara Jarzab ${ }^{1 *}$

\begin{abstract}
Background: Differential diagnosis between malignant follicular thyroid cancer (FTC) and benign follicular thyroid adenoma (FTA) is a great challenge for even an experienced pathologist and requires special effort. Molecular markers may potentially support a differential diagnosis between FTC and FTA in postoperative specimens. The purpose of this study was to derive molecular support for differential post-operative diagnosis, in the form of a simple multigene mRNA-based classifier that would differentiate between FTC and FTA tissue samples.
\end{abstract}

Methods: A molecular classifier was created based on a combined analysis of two microarray datasets (using 66 thyroid samples). The performance of the classifier was assessed using an independent dataset comprising 71 formalin-fixed paraffin-embedded (FFPE) samples (31 FTC and 40 FTA), which were analysed by quantitative real-time PCR ( $q P C R$ ). In addition, three other microarray datasets (62 samples) were used to confirm the utility of the classifier.

Results: Five of 8 genes selected from training datasets (ELMO1, EMCN, ITIH5, KCNAB1, SLCO2A1) were amplified by qPCR in FFPE material from an independent sample set. Three other genes did not amplify in FFPE material, probably due to low abundance. All 5 analysed genes were downregulated in FTC compared to FTA. The sensitivity and specificity of the 5-gene classifier tested on the FFPE dataset were $71 \%$ and $72 \%$, respectively.

Conclusions: The proposed approach could support histopathological examination: 5-gene classifier may aid in molecular discrimination between FTC and FTA in FFPE material.

Keywords: Follicular thyroid adenoma, Follicular thyroid cancer, Gene expression, Microarray, Formalin-fixed paraffin-embedded blocks

\footnotetext{
*Correspondence: bjarzab@io.gliwice.pl

${ }^{\dagger}$ Equal contributors

'Department of Nuclear Medicine and Endocrine Oncology, Maria

Skłodowska-Curie Memorial Cancer Center and Institute of Oncology, Gliwice

Branch, Wybrzeże Armii Krajowej 15, Gliwice 44-101, Poland

Full list of author information is available at the end of the article
} 


\section{Background}

Discrimination between malignant follicular thyroid cancer (FTC) and benign follicular thyroid adenoma (FTA) is the most difficult aspect of thyroid pathology. Postsurgical (post-thyroid unilateral lobectomy) FTC and FTA management algorithms are different: only cancer patients require completion total thyroidectomy, adjuvant radioiodine treatment, and long-term follow-up. The histological diagnosis of FTC remains a challenge for pathologists, as the diagnostic criteria of FTC, namely capsular invasion or angioinvasion, are prone to serious inter-observer variability [1]. Thus, the discrimination of FTC from FTA is an important clinical problem, particularly for minimally invasive cases, and depends on the number of serial sections and tumour regions examined [2].

Several mutations play an important role in the biology of follicular tumours, such as paired box gene 8 (PAX8)/ peroxisome proliferator-activated receptor gamma (PPARG) translocation and $R A S$ point mutations. The first one occurs in $35-47 \%$ of FTC and up to $13 \%$ of FTA [3-5], the second occurs in approximately $20-50 \%$ and $19 \%$ of FTC and FTA, respectively [6-9].

Numerous single immunohistochemical markers have been proposed, and the most widely accepted single protein that improves diagnostic accuracy is galectin 3, even in the case of minimally invasive follicular carcinoma [10]. Obviously, immunohistochemical panels can be extended by including other proteins e.g., cytokeratin 19 or p27 [11]. Many other potential markers have been considered, such as HBME-1, extracellular matrix metalloproteinase inducer (EMMPRIN), growth arrest and DNA damage-inducible gene 153 (GADD153), thyroid transcription factor 1 (TFF-1), Ki-67, p63, and p53 [12-15], but the conclusions are limited by the relatively small size of tested populations.

Alternatively, the FTC and FTA differentiation problem was investigated in several miRNA-profiling studies. Some miRNAs are described as sensitive biomarkers of malignant and benign follicular thyroid tumours [16-18], but the overlap of differentiating miRNAs pointed out in these studies is limited.

A number of attempts have been made to improve the molecular diagnosis of FTC using specific mRNA signatures of malignant follicular tumours [19-26]. The most promising of these, by Borup and co-workers [19] was based on a 40-sample microarray dataset and led to the delineation of a 76-gene signature, which was highly sensitive and specific both for their own dataset and when tested with 2 previously published microarray datasets $[21,24]$. Simultaneously, a recent publication by Chudova et al. proposed a 167-gene classifier that was able to diagnose thyroid nodules with indeterminate cytology [27]. Although the classifier was trained on various types of thyroid benign and malignant nodules, the prospective multicentre validation study showed good specificity for follicular thyroid nodules [28]. The proposed predictors might be an important step to increase the effectiveness of thyroid nodule diagnosis. The vast majority of recent studies have concentrated on preoperative differential diagnosis [26,28], with molecular tests applied to freshfrozen material with an aim to translate it into fine-needle aspiration biopsy specimen testing. This is evidently very important in the context of preoperative diagnosis, but it may not be the most efficient method for direct application to the analysis of formalin-fixed paraffin-embedded (FFPE) samples at the mRNA level, which in our opinion may aid postoperative differential diagnosis in controversial cases. In this study, we aimed to combine the gathered knowledge about the transcriptomes of FTC and FTA to derive a novel classifier of thyroid follicular malignancy applicable to FFPE material.

We therefore used the aforementioned available dataset of Borup et al. for gene pre-selection, further selected genes and trained the classifier on very carefully selected FTC and FTA samples, and verified the classifier by real-time quantitative PCR (qPCR) analysis in formalin-fixed paraffinembedded (FFPE) samples.

\section{Methods}

\section{Patients}

Tumour samples for microarray analysis (fresh-frozen [FF] material) were derived from 27 FTC (median age, 68 years) and 25 FTA (median age, 47 years) patients treated by thyroidectomy in Polish and German centers. The study was approved by the local ethics committees of the MSC Memorial Cancer Center and Institute of Oncology (Gliwice, Poland), University of Leipzig (Leipzig, Germany), University of Halle (Halle, Germany), and Mainz University Hospital (Mainz, Germany) and informed consent was obtained from all the patients.

Tumour samples for further validation (FFPE blocks) were derived from 31 FTC patients (median age of patients, 61 years) and 40 FTA patients (median age, 49 years). All patients were treated by thyroid surgery at MSC Memorial Cancer Center and Institute of Oncology, Gliwice Branch, and samples were subjected to routine histopathological examination at the Department of Pathology between 2006 and 2010.

\section{Microarray analysis}

To obtain the highest possible level of adequacy for histopathological diagnosis, samples containing enough tissue material were subjected to independent and blinded review by 2 thyroid pathology experts (S.H. and D.L.). Only samples with full assessment and concordant diagnoses from both pathologists were selected for the training dataset (hereafter "training dataset B"), which included 13 FTC and 13 FTA samples. The remaining samples were used as an independent set of samples 
(14 FTC and 12 FTA samples) constituting testing dataset D (Table 1), in which the initial clinical diagnosis was used to describe the sample. The term "training dataset" indicates the sample group used to build the gene classifier and the term "testing dataset" indicates the sample group used to test its performance.

RNA for microarray analysis was isolated using the RNeasy Mini kit (Qiagen, Hilden, Germany) after tumour content verification of the specimen by a pathologist. The standard Affymetrix microarray protocol was carried out, and samples were hybridized with the HG-U133 Plus 2.0 microarray. Microarray data analysis was performed in an R/Bioconductor environment. Datasets were pre-processed using the GC Robust Multiarray Average (GCRMA) method [29]. The classifier was developed using the CMA package [30]. Detailed methods are described in supplemental information (Additional file 1).

\section{Validation experiment in FFPE samples}

Blocks with sufficient tumour tissue in the specimen (approximately 80\%) were selected. RNA was isolated using the FFPE RNeasy Mini Kit (Qiagen) from 5 slices of paraffin blocks selected by a histopathologist. Details are provided in Additional file 1.

Real-time quantitative PCR (qPCR) was carried out for 8 genes selected from the training dataset: carbonic anhydrase IV (CA4), engulfment and cell motility 1 (ELMO1), endomucin (EMCN), inter-alpha-trypsin inhibitor heavy chain family, member 5 (ITIH5), potassium voltagegated channel, shaker-related subfamily, beta member 1 (KCNAB1), low density lipoprotein receptor-related protein $1 \mathrm{~B}(L R P 1 B)$, pleckstrin homology domain containing, family G (with RhoGef domain) member 4B (PLEKHG4B), and solute carrier organic anion transporter family, member 2A1 (SLCO2A1). PCR amplification was performed with Universal Probe Library fluorescent probes (Roche, Basel, Switzerland) and a $5^{\prime}$-nuclease assay, starting with 200 ng of total RNA. Normalization was carried out in

Table 1 Description of datasets analyzed in the study (see Additional file 1 for detailed information)

\begin{tabular}{lccc}
\hline Dataset & $\begin{array}{c}\text { FTC } \\
\text { samples }\end{array}$ & $\begin{array}{c}\text { FTA } \\
\text { samples }\end{array}$ & Ref. \\
\hline Borup et al. microarray dataset A & 18 & 22 & {$[19]$} \\
Training microarray dataset B & 13 & 13 & this study \\
Validation qPCR dataset C & 31 & 40 & this study \\
Validation microarray dataset D & 14 & 12 & this study \\
$\begin{array}{l}\text { Weber et al. validation microarray } \\
\text { dataset E1 }\end{array}$ & 12 & 12 & [24] \\
Hinsch et al. validation microarray & 8 & 4 & [21] \\
dataset E2 & & & \\
Total & 96 & 103 & \\
\hline
\end{tabular}

the GeNorm application [31]. Details of the methods are provided in Additional file 1.

\section{External microarray data and analysis pipeline}

Three microarray datasets were downloaded from gene expression repositories: the datasets of Borup et al. (dataset A, 22 FTA and 18 FTC), Weber et al. (testing dataset E1, 12 FTA and 12 FTC), and Hinsch et al. (testing dataset E2, 4 FTA and 8 FTC). Details are provided in Table 1 and in Additional file 1.

Dataset A was initially used for gene pre-selection. Further selection of genes and training of the classifier was carried out on dataset B (13 FTA and 13 FTC). The obtained classifier was tested in a group of independent new FFPE samples analyzed by qPCR (dataset C, 40 FTA and 31 FTC). Additional testing was carried out on microarray datasets D (our own samples, fresh-frozen, 12 FTA and 14 FTC) and the publicly available microarray datasets E1 and E2. In total, 199 thyroid samples were analyzed (123 of our own samples and 76 publicly available samples). The clinical characteristics of all specimens used in the analysis is presented in Table 1 and the analysis pipeline is described in Figure 1.

\section{Classifier construction and validation}

Genes that exhibited significant differences between FTC and FTA were pre-selected from dataset A. The selection criteria were as follows: Student's t-test (with equal variances assumed), non-corrected p-value $<0.0005(<0.09$ when the p-value was corrected for multiple comparisons using the false discovery rate method), mean gene expression $>5$ in either the FTC or FTA group, and an absolute log ratio between the groups $>1.5$. Transcripts not fully annotated were filtered out. When more than one probe set per transcript was found, the probe set with the lowest p-value was selected.

Based on the subset of pre-selected genes, further gene selection using the Student's t-test (equal variances assumed), and classifier training were carried out on dataset $B$. Diagonal linear discriminant analysis (DLDA), a simple and reliable method based on linear combinations of genes, was chosen as the classification engine. However, other more sophisticated methods of classification were also used for comparison. Details of the analysis are provided in Additional file 1. The accuracy of the classification was assessed on dataset B using 10-fold cross-validation, repeated 10 times.

The 5 -gene classifier was validated by qPCR on dataset $\mathrm{C}$. First, the normalized expression data were $\log _{2}$-transformed. Then, the classifier performance was assessed using leaveone-out cross-validation (LOOCV; in each iteration, the DLDA classifier was trained on $\mathrm{n}-1$ samples and tested on the remaining one). All 5 genes (validated by qPCR, details in results) from dataset $C$ were used in each iteration 


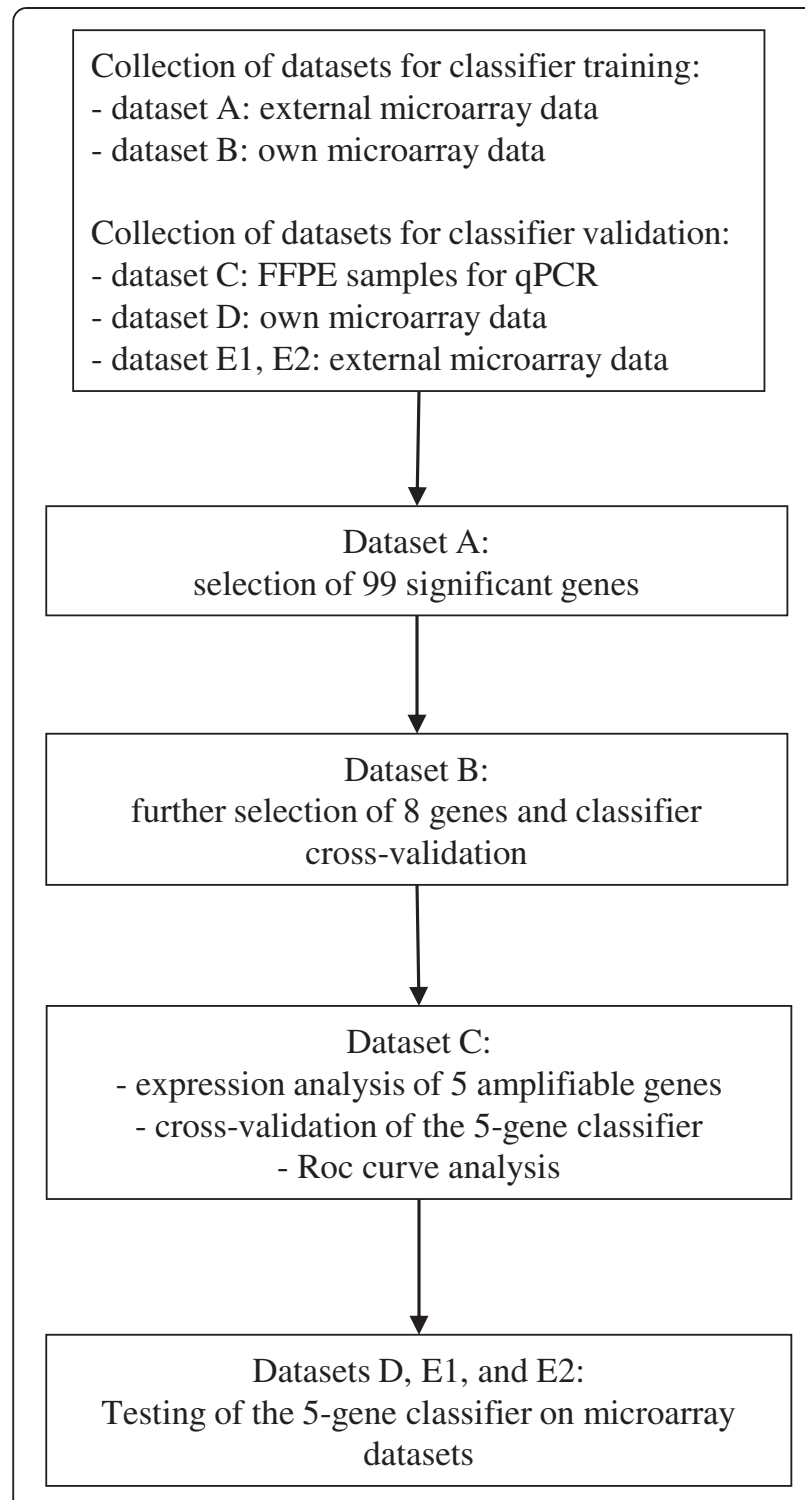

Figure 1 Analysis pipeline. First, datasets were collected. Second, the micorarray dataset A was analyzed and 99 genes were selected. Third, the microarray dataset B was analyzed for further selection of 8 genes and classifier cross-validation. Next, qPCR dataset $C$ was analyzed in order to validate the classifier. Finally, public datasets $D$, E1 and E2 were analysed to test the classifier.

and no further gene selection was used in the crossvalidation loops.

Dataset $\mathrm{C}$ was also used to create a receiver operating characteristic (ROC) curve and to assess the diagnostic efficacy of the classifier. In the leave-one-out loop, for each sample, we calculated the probability that the sample belongs to the FTC class. Varying the threshold for the probability, the ROC curve was plotted.

An additional validation of the 5-gene classifier was performed using microarray data (datasets D, E1, E2). Both training dataset $\mathrm{B}$ and testing dataset $\mathrm{D}$ contained microarrays of the same type. Therefore, the final classifier, which had been trained on dataset B, was directly tested on dataset $\mathrm{D}$. This approach was not possible for testing datasets E1 and E2 as they contained different microarray types. Therefore, LOOCV was used to assess the accuracy of the classifier on those 2 datasets. In all validation steps, only 5 genes amplified by qPCR were analyzed in the microarray test datasets and no further gene selection was used in the cross-validation loops.

\section{Comparison of the classifiers}

To compare the 5-gene classifier developed by us to other published classifiers (the 76-gene classifier developed by Borup et al. [19], 3-gene classifier developed by Weber et al. [24], and 5-gene classifier developed by Foukakis et al. [32]), we calculated the accuracies of those classifiers in 4 different datasets: dataset B (our own), dataset D (our own), dataset E1 [24], and dataset E2 [21].

Borup's classifier was originally created on dataset A and was based on 76 differentially expressed genes and the SVM method with a radial kernel. To calculate its accuracy on dataset $\mathrm{B}$, we performed a 10 -fold cross-validation of such a classifier with a fixed list of 76 genes. To calculate its accuracy on dataset $\mathrm{D}$, we trained this classifier on dataset $\mathrm{B}$ and tested it on dataset $\mathrm{D}$. The accuracy of this classifier on datasets E1 and E2 had already been reported by Borup et al. and we used those values for comparison.

To calculate the accuracies of the classifiers developed by Weber and Foukakis, we applied a DLDA classification method. Analogous to the analysis above, to calculate the accuracies of these classifiers on dataset B, we performed a 10 -fold cross-validation. To calculate their accuracies on dataset $\mathrm{D}$, we trained those classifiers on dataset $\mathrm{B}$ and tested on dataset D. To calculate their accuracies on dataset E1 and E2, we performed LOOCV analysis.

The accuracy of the 5-gene DLDA classifier on dataset $B$ was calculated by 10 -fold cross-validation (genes were selected by t-test). The calculation of the accuracy of our classifier on datasets D, E1, and E2 is described above.

\section{Results}

\section{Developing a robust classifier for FTC and FTA} differentiation

Because we aimed to obtain the most robust classifier possible, and had access to 2 reliable datasets (our own dataset with 26 samples [training dataset $B$ ] and the dataset of Borup et al. with 40 samples [dataset A]), we decided to use both of them sequentially for classifier construction. First, from dataset A (large enough to represent the variability of FTC) we selected 99 genes with high significance and large magnitude of difference (see Methods; the genes are listed in Additional file 2, and the raw expression values of these genes in dataset $B$ are attached in Additional file 3). Second, based on 
the pre-filtered genes, we used dataset B (with histological diagnosis verification for each sample, based on the consensus diagnosis of 2 independent histopathologists) to build the classifier.

Cross-validation revealed that for the majority of classification engines used, there was no meaningful increase in multigene classifier accuracy, when more than 20 genes were used within the preselected dataset of 99 genes. (Additional file 1: Figure A). In an attempt to create a classifier of low complexity and due to the material limitation, we decided to validate only 8 genes, which provided almost the same accuracy as that for a larger number of genes: $80 \%$ and $84 \%$ accuracies for the 8-gene and 45-gene classifiers (the one with maximal accuracy), respectively (Table 2).

For the classifier, we selected 8 transcripts that were most significant in the analysis of 99 preselected genes on dataset B: CA4, ELMO1, EMCN, ITIH5, KCNAB1, $L R P 1 B, P L E K H G 4 B$, and SLCO2A1. The classifier built on these genes was referred to as the 8-gene classifier. Although we did not use information regarding the direction of the gene expression change, all of these most significant genes were downregulated in FTC. Detailed information about the significance of these genes in all the datasets used in the paper is included in Additional file 4.

\section{Classifier testing on FFPE material and qPCR}

We assessed the expression of all the 8 selected genes in serial dilutions of calibrator RNA (mixture of excellent quality RNA from FTA and FTC). Because of the poor amplification of $L R P 1 B$, the gene was excluded from further analysis (insufficient qPCR efficiency). Next, the genes of the 8-gene classifier were analyzed in test dataset $\mathrm{C}$ (40 FTA and 31 FTC samples, RNA from FFPE blocks). CA4 and PLEKHGAB did not amplify sufficiently in FFPE samples (CA4 was amplified in 4 FTA and none of the FTC samples, and PLEKHG4B in 3 FTAs and 1 FTC samples; data not shown). This was probably due to the low constitutive expression of these genes or their high susceptibility to degradation (Additional file 1: Figure C). To verify this, we evaluated the expression of these genes by transcriptome sequencing of 2 FTCs and noted that $L R P 1 B, C A 4$, and PLEKHGAB exhibited low abundance in FTC, compared to other genes (Additional file 1: Figure D). Considering the poor amplification of the 3 genes, we decided to proceed with the 5-gene classifier.

We confirmed that ELMO1, EMCN, ITIH5, KCNAB1, and SLCO2A1 were downregulated in follicular carcinoma compared to adenoma (Figure 2). The statistical significance of the differences for all 5 transcripts was assessed using the Mann-Whitney test. The results were significant for all of them when no correction for multiple comparison was used (p-value < 0.05), and for 4 of them (except ITIH5) when the Bonferroni correction was used (Table 3).

We tested the final 5-gene classifier on the qPCR/FFPEderived test dataset $\mathrm{C}$ using a cross-validation approach and obtained a classification accuracy of $72 \%$, sensitivity of $72 \%$, specificity of $71 \%$, positive predictive value (PPV) of $72 \%$, and negative predictive value (NPV) of 67\% (Table 2). Given that both PPV and NPV depend on the composition of the dataset, we also calculated the positive and negative likelihood ratios, which were 2.58 and 0.4 , respectively.

Table 2 Performance measures of classifiers in different datasets

\begin{tabular}{|c|c|c|c|c|c|c|c|}
\hline $\begin{array}{l}\text { Dataset (origin, } \\
\text { method of analysis) }\end{array}$ & Method & Accuracy (\%) & PPV (\%) & NPV (\%) & Sensitivity (\%) & Specificity (\%) & $\begin{array}{l}\text { Prevalence } \\
\text { of FTC in } \\
\text { dataset (\%) }\end{array}$ \\
\hline \multirow[t]{2}{*}{ B (own, microarray) } & $\begin{array}{l}\text { DLDA classification based on } \\
\text { the } 8 \text { best genes chosen from } \\
99 \text { preselected ones.* }\end{array}$ & 80 & 82 & 78 & 76 & 83 & 50 \\
\hline & $\begin{array}{l}\text { DLDA classification based on } \\
45 \text { (optimal number) best } \\
\text { genes chosen from } 99 \\
\text { preselected ones.* }\end{array}$ & 84 & 85 & 83 & 83 & 85 & 50 \\
\hline \multirow[t]{2}{*}{$C$ (own, FFPE qPCR) } & $\begin{array}{l}\text { 5-gene DLDA classification } \\
\text { (cut-off } 0.5)^{* *}\end{array}$ & $\begin{array}{l}\text { 72(95\% Cl: } \\
60-82)\end{array}$ & $\begin{array}{l}\text { 67(95\% Cl: } \\
48-82)\end{array}$ & $\begin{array}{l}76(95 \% \mathrm{Cl}: \\
60-89)\end{array}$ & $\begin{array}{l}71(95 \% \mathrm{Cl}: \\
52-86)\end{array}$ & $\begin{array}{l}\text { 72(95\% Cl: } \\
56-85)\end{array}$ & 44 \\
\hline & $\begin{array}{l}\text { 5-gene DLDA classification } \\
\text { (cut-off } 0.12)^{* *}\end{array}$ & 70 & 61 & 88 & 90 & 55 & 44 \\
\hline D (own, microarray) & $\begin{array}{l}\text { 5-gene DLDA classifier trained } \\
\text { on dataset B, tested on D }\end{array}$ & 73 & 77 & 69 & 71 & 75 & 54 \\
\hline $\begin{array}{l}\text { E1 (Weber et al. } \\
\text { microarray) }\end{array}$ & 5-gene DLDA classifier.** & 92 & 100 & 86 & 83 & 100 & 50 \\
\hline $\begin{array}{l}\text { E2 (Hinsch et al. } \\
\text { microarray) }\end{array}$ & 5-gene DLDA classifier.** & 83 & 100 & 67 & 75 & 100 & 67 \\
\hline
\end{tabular}

Accuracy, proportion of all samples that are correctly classified; $P P V$, positive predictive value; NPV, negative predictive value; SVM, support vector machines; DLDA, diagonal linear discriminant analysis; $\mathrm{Cl}$, confidence interval. *Performance assessed by 10 -fold cross-validation. **Performance assessed by leave-one-out cross-validation. 

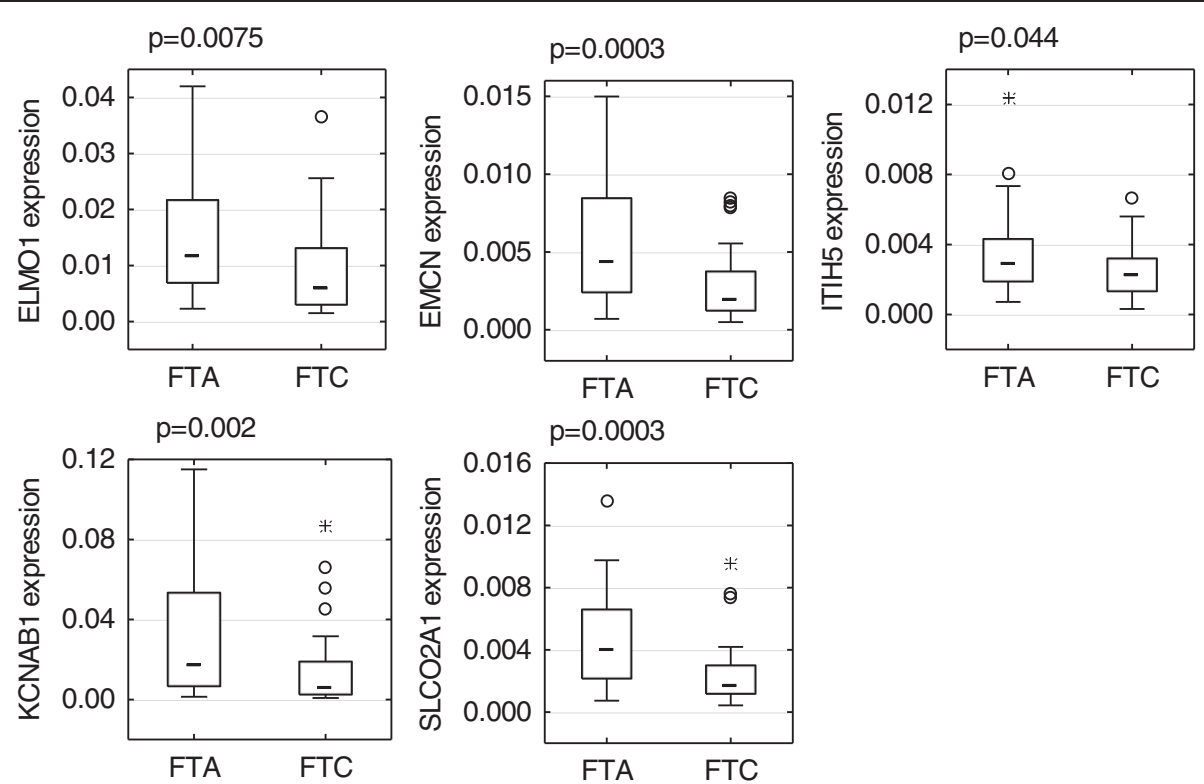

Figure 2 Boxplots for the validated genes (ELMO1, EMCN, ITIH5, KCNAB1, and SLCO2A1). All genes were under-expressed in follicular thyroid carcinoma (FTC) compared to follicular thyroid adenoma (FTA). All p-values were calculated using Mann-Whitney $U$ test. The boxplots show following values: median: middle line; 25-75 percentile: box; non-outlying range: whiskers; outliers: circles; extreme values: stars.

In our analysis, we treated the sensitivity and specificity as equally important. For diagnostic selection applications, however, maximizing sensitivity could be of greater importance, even if that decreases specificity. To analyse this aspect in-depth, we used the ROC curve. By shifting the 5 -gene classifier probability threshold from 0.50 to 0.12 , we were able to achieve a sensitivity of $90 \%$, with some decrease in specificity $(55 \%)$, and in the overall accuracy of the test (70\%) (Table 2, Figure 3).

\section{Classifier testing on microarray datasets}

The 5-gene classifier was further tested on 3 microarray datasets: D, E1, and E2. The classifier was trained on dataset
$\mathrm{B}$ and tested on dataset $\mathrm{D}$, resulting in an accuracy of $73 \%$. Cross-validation of the classifier on datasets E1 and E2 provided accuracies of $92 \%$ and $83 \%$, respectively (Table 2). These results further confirmed the reliability of the classifier.

\section{Comparison of the classifiers}

We compared our simple 5-gene signature developed in combined analysis of dataset A and B to the complex 76-gene signature developed by Borup et al. on dataset A. We also compared our signature to the other classifiers, which are also composed of a small number of genes $[24,32]$. The accuracies of these classifiers, calculated on 4 datasets, are included in Table 4.

Table 3 Results for the $\mathbf{8}$ genes included in the classifier and chosen for qPCR validation on FFPE samples

\begin{tabular}{lcccccc}
\hline Gene & \multicolumn{3}{c}{ Signal log ratio (FTC vs. FTA) } & & Non-corrected p-value & Bonferroni-corrected p-value \\
\cline { 2 - 4 } & Dataset A & Training dataset B & Validation dataset C (FFPE) & & Validation dataset C (FFPE) & Validation dataset C (FFPE) \\
\hline ELMO1 & -2.15 & -2.36 & -0.98 & $\mathbf{0 . 0 0 7 5}$ & 0.0377 \\
EMCN & -1.94 & -2.25 & -1.17 & $\mathbf{0 . 0 0 0 3}$ & 0.0015 \\
ITIH5 & -1.51 & -1.98 & -0.38 & $\mathbf{0 . 0 4 4 3}$ & 0.2214 \\
KCNAB1 & -2.45 & -3.35 & -1.67 & $\mathbf{0 . 0 0 2 0}$ & 0.0100 \\
SLCO2A1 & -1.86 & -1.45 & -1.23 & $\mathbf{0 . 0 0 0 3}$ & 0.0014 \\
LRP1B & -2.15 & -2.7 & & Insufficient amplification & \\
CA4 & -2.77 & -2.92 & & Insufficient amplification & \\
PLEKHG4B & -1.88 & -1.36 & Insufficient amplification &
\end{tabular}

Log ratios of the 8 genes from microarray training datasets $A$ and $B$, and validation dataset $C$ (qPCR), are presented. Mann-Whitney $U$ test results for genes validated with qPCR are in bold. 


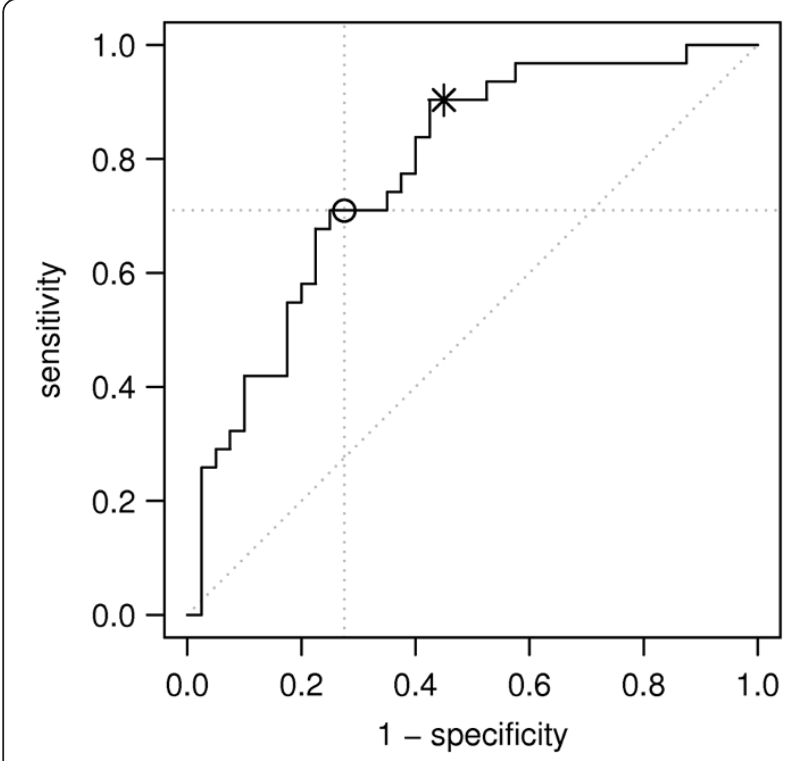

Figure 3 ROC curve for the DLDA classifier that was

cross-validated on the dataset $\mathbf{C}$. The circle marks the classifier with a cut-off of 0.5 (specificity $=71 \%$, sensitivity $=72 \%$ ). The star marks the classifier with a cut-off of 0.12 (specificity, $90 \%$; sensitivity, 55\%).

\section{Discussion}

Although follicular tumours are routinely diagnosed post-surgically by classic histological criteria (tumour capsule infiltration and/or angioinvasion), this field of pathology is facing numerous challenges. These problems were recognized early but are still not fully resolved. A study from 1978 in Scandinavia has shown observer disagreement of nearly $30 \%$ when examining follicular carcinoma [33]. A report of agreement between 5 pathologists diagnosing follicular thyroid tumours reported their final consensus diagnosis in the range of 0.11-0.69 [1]. The inter-observer agreement for FTC diagnosis in that paper was estimated to 0.23 , with large intra-observer variability (0.68) [1]. Similar data were obtained by our group [34]. This stresses the need for extensive training of pathologists involved in the diagnosis of follicular tumours, and the importance of reference centers, but also points to the necessity for tools that can improve pathologist accuracy. When mining for novel molecular markers, it is important that a consensus diagnosis by experienced pathologists be used, as was done in our work.
A number of gene expression profiling studies have attempted to identify transcripts that are differentially expressed between FTA and FTC [19-25]. However, none of these studies resulted in a simple, efficient gene signature that was applicable in clinical practice.

Recently, a promising gene expression classifier based on 167 genes was created [27] and validated [28] in a large multicentre trial. It was used for the discrimination between benign and malignant thyroid nodules that cannot be determined by cytology. It achieved a sensitivity of $92 \%$ and specificity of $52 \%$, which is similar to our results (sensitivity $90 \%$ and specificity $55 \%$ ). While these studies as well as other ones $[28,35]$ lead towards a classifier applicable to fine-needle aspiration biopsy material, our approach is, in fact, different from these other published reports. We aim to support the diagnosis of post-thyroidectomy FFPE material carried out by routine histopathology with additional mRNA-based markers. Such a tool could be used as a pre-selection test to identify tumours that need meticulous histopathological evaluation. The genes selected in our study are differentiating in degraded mRNA specimens from FFPE blocks, and thus might potentially serve as a molecular indicator of malignancy. Both approaches (preoperative small sample cytology molecular testing [28] and post-operative whole section FFPE-based analysis, as proposed here) are in fact complementary and might be applied sequentially to provide the optimal final diagnosis.

In our opinion, it is necessary that the classifier be limited in the terms of genes tested, as cost-effectiveness issues may hamper the clinical application of multigene signatures; thus, selecting the optimal panel of markers for further testing is of utmost importance, especially from the perspective of health systems not reimbursing the cost of complex genomic diagnostic methods.

One of the important limitations of many previous studies concerning FTC/FTA differences is their relatively small sample size. To our knowledge, in all microarray studies published before 2010, the number of follicular tumours ranged from 7 to 28 [20,22]. The Borup et al. study, published in 2010, was based on a dataset of 40 follicular tumours, the largest non-custom microarray dataset of follicular tumours to date. Thus, the gene preselection in our study was based on a re-analysis of the Borup dataset.

Table 4 Accuracy comparison for various classifiers

\begin{tabular}{lllll}
\hline Classifier description & Accuracy on dataset B & Accuracy on dataset D & Accuracy on dataset E1 & Accuracy on dataset E2 \\
\hline 76-gene Borup's classifier & $81 \%$ & $81 \%$ & $92 \%$ & $83 \%$ \\
5-gene own classifier & $77 \%$ & $73 \%$ & $92 \%$ & $83 \%$ \\
3-gene Weber's classifier & $77 \%$ & $50 \%$ & $91 \% *$ & $42 \%$ \\
5-gene Foukakis classifier & $77 \%$ & $61 \%$ & $71 \%$ & $75 \%$
\end{tabular}

*Potentially overfitting (tested on the set from which it was developed). 
Although our final 5-gene classifier is much smaller, it exhibits accuracy comparable to that obtained using the large, 76-gene classifier of Borup et al. On 2 of 4 datasets used for comparison, it gave the same accuracy as the large classifier, and in the other 2, the loss of accuracy was lower than $10 \%$. When compared to other classifiers with a similar number of genes (3-gene classifier of Weber and 5-gene classifier of Foukakis), our classifier gives better accuracies on validation datasets. Our test set (dataset C), derived from FFPE samples and analyzed using qPCR, reproduces cases obtained in a routine setting. The classifier accuracy obtained on this dataset is $72 \%$, with a sensitivity of $71 \%$ and specificity of $72 \%$. For the detection of cancer, the sensitivity is of utmost importance; shifting the test threshold gives a sensitivity of $90 \%$, with an acceptable specificity of $55 \%$. This approach seems justified, as the gain in FTC diagnosis sensitivity would outweigh the drop in specificity via careful histopathological assessment.

From the initial 8-gene classifier, 3 genes: $C A 4, L R P 1 B$, and PLEKHG $4 B$, could not be amplified with qPCR, probably due to their low expression in FFPE specimens. The remaining 5 genes, positively validated in our analysis, are downregulated in FTC compared to adenoma. ELMO1, EMCN, KCNAB1, and SLCO2A1 are plasma membrane components and ITIH5 is an extracellular matrix (ECM) component. Some of these proteins exhibit functional similarities: SLCO2A1 and KCNAB1 exhibit transporter activity [36,37], and ELMO1, EMCN, and ITIH5 are involved in cell movement and ECM stability [38-40]. Some of the genes from our 5-gene classifier have already been mentioned in other studies of high-throughput gene expression analysis of follicular tumours. ITIH5, KCNAB1, and $S L C O 2 A 1$ are mentioned in the Borup et al. study. Besides, ELMO1 is differentially expressed in Barden et al. [41] and KCNAB1 is differentially expressed in 2 other papers by Takano [23] and Weber [24], respectively.

There are some potential limitations to our study. The most important issue, applying both to our analysis as well to all other studies carried out to date, is that we do not understand the reason for sample misclassification. Are the misclassified samples the same that would be misclassified by histopathologists? The excellent prognosis of follicular cancer treated with adequate surgical and adjuvant therapy makes this assessment difficult, given that patients exhibiting disease recurrence or dissemination initially or during follow up are relatively rare. This issue will be addressed in future studies, preferably using gene expression profiling of FTCs with metastases or local recurrence. We also cannot exclude the possibility that the reason for incorrect classification of some samples is that the signal from a small amount of neoplastic tissue might be dominated by the surrounding normal thyroid tissue.

Because routine diagnostic applications of the test is our goal, we did not apply any methods, such as paraffin slide micro dissection, to enrich the tumour content of our samples, as these methods are difficult to apply in the clinical setting. We believe that our signature is useful, as it was constructed using 2 large datasets and validated on an independent dataset using a different method. Although it is not efficient enough to be used as a clinical diagnostic test by itself, it would improve the process of diagnosis [42]. It is also a step towards developing a highly powerful classifier, as each microarray dataset generated and analyzed improves our understanding of gene expression differences between different types of follicular tumours. Additional studies also should be undertaken to elucidate the molecular and clinical aspects of the discovered markers.

We must stress that the problem of FTC and FTA differentiation has also been investigated by several miRNA-profiling studies. miRNAs are known to be a good disease markers, and can be easily detected in frozen tissues, paraffin blocks (FFPE), fine-needle aspiration biopsies (FNAB), or even serum [43]. Some miRNAs are described as sensitive biomarkers of malignant and benign follicular thyroid tumours [16-18]. In future, a possible FNA or FFPE-block test might combine degradationresistant mRNA markers or protein markers and a panel of miRNAs to provide optimal analytical parameters.

\section{Conclusions}

In summary, we developed a simple 5-gene classifier that can distinguish FTC from FTA with good accuracy. It is based on the 2 largest FTC-FTA microarray datasets and was validated on an independent FFPE sample set, with a potential sensitivity of up to $90 \%$. In future, such a molecular test may serve as an important tool for assisting pathologists in cases of thyroid follicular neoplasms where a clear clinical decision cannot be made based on histopathology.

\section{Additional files}

Additional file 1: Description of data: This file contains additional detailed descriptions of Materials, Methods, and Results.

Additional file 2: Description of genes differentially expressed between FTC and FTA, derived in the analysis of dataset A. Description of data: The table contains the list of 99 genes that exhibited significant difference between FTC and FTA in the analysis of dataset A. The table contains gene annotation information (affyids, symbols, names), analysis results (p-values and log-ratios calculated in datasets A and B), and information pertaining to which of the genes belong to the 5-gene, 8-gene, and optimal 45-gene classifiers.

Additional file 3: Expression values of genes differentially expressed between FTC and FTA, derived in the analysis of dataset A. Description of data: The table contains the expression values of 99 genes that exhibited significant differences between FTC and FTA samples in the analysis of dataset A. The table presents their expression values in samples belonging to dataset $\mathrm{B}$.

Additional file 4: Significance of the 8 selected genes in all analyzed datasets. Description of data: The table contains the results of differential analysis for 8 selected genes in all analyzed datasets: A, B, C, $D, E 1$, and E2. For each dataset and gene, it contains the p-value, fold change, log ratio, and the rank (according to the $p$-value) of the gene. 


\section{Abbreviations}

FTC: Follicular thyroid cancer; FTA: Follicular thyroid adenoma; qPCR: Quantitative real-time PCR; FNAB: Fine needle aspiration biopsy; FFPE: Formalin-fixed paraffin-embedded; FF: Fresh-frozen; GCRMA: GC robust multiarray average; DLDA: Diagonal linear discriminant analysis; LOOCV: Leave-one-out cross-validation; CA4: Carbonic anhydrase IV; ELMO1: Engulfment and cell motility 1; EMCN: Endomucin; ITIH5: Inter-alpha-trypsin inhibitor heavy chain family, member 5; KCNAB1: Potassium voltage-gated channel, shaker-related subfamily, beta member 1; LRP1B: Low density lipoprotein receptor-related protein $1 \mathrm{~B}$; PLEKHG4B: pleckstrin homology domain containing, family G (with RhoGef domain) member 4B; SLCO2A1: Solute carrier organic anion transporter family, member 2A1; PPV: Positive predictive value; NPV: Negative predictive value; ROC: Receiver operating characteristic; ECM: Extracellular matrix; PPARG: Peroxisome proliferator-activated receptor gamma; PDTC: Poorly differentiated thyroid carcinoma; PAX8: Paired box gene 8; EMMPRIN: Extracellular matrix metalloproteinase inducer; GADD153: Growth arrest- and DNA damage-inducible gene 153; TFF-1: Thyroid transcription factor 1 .

\section{Competing interests}

The authors declare that they have no competing interests.

\section{Authors' contributions}

$A P, B W, M J, B J$ contributed to the writing of the manuscript. MJ, ME, RP, and $B J$ designed and coordinated the study. AC and TM collected the tissue material. AK analysed patient data. ES, SH, and DL performed the histopathological examination of the samples. BW, MOW, DR, TT, MK performed the experiments described in this study. AP, TS and MS performed the bioinformatics analysis. RP and ME made critical revisions. All authors have read and approved the final manuscript.

\section{Acknowledgements}

We thank Dr. Krzysztof Fujarewicz for critically analysing the manuscript and for his helpful suggestions. This work was supported by the Ministry of Science and Higher Education grants nr N N401 072637, N N403 194340 and Foundation for Polish Science MPD Program "Molecular Genomics, Transcriptomics and Bioinformatics in Cancer" and Postgraduate School of Molecular Medicine (BW and TS). This research was also supported by a DFG grant to Markus Eszlinger (ES162/4-1). Author of this publication (AP) is a scholar in SWIFT project POKL.08.02.01-24-005/10, which is co-financed by European Union within European Social Fund.

\section{Author details}

${ }^{1}$ Department of Nuclear Medicine and Endocrine Oncology, Maria Skłodowska-Curie Memorial Cancer Center and Institute of Oncology, Gliwice Branch, Wybrzeże Armii Krajowej 15, Gliwice 44-101, Poland. ${ }^{2}$ Clinic of Oncological and Reconstructive Surgery, Maria Skłodowska-Curie Memorial Cancer Center and Institute of Oncology, Gliwice Branch, Wybrzeże Armii Krajowej 15, Gliwice 44-101, Poland. ${ }^{3}$ Radiotherapy and Chemotherapy Clinic, Maria Skłodowska-Curie Memorial Cancer Center and Institute of Oncology, Gliwice Branch, Wybrzeże Armii Krajowej 15, Gliwice 44-101, Poland. ${ }^{4}$ Tumor Pathology Department, Maria Skłodowska-Curie Memorial Cancer Center and Institute of Oncology, Gliwice Branch, Wybrzeże Armii Krajowej 15, Gliwice 44-101, Poland. ${ }^{5}$ Faculty of Automatic Control, Electronics and Computer Science, Silesian University of Technology, Gliwice, Poland. ${ }^{6}$ Division of Endocrinology and Nephrology, University of Leipzig, Leipzig, Germany. ${ }^{7}$ Department of General, Visceral, and Transplantation Surgery, University Medical Center of the Johannes Gutenberg University, Mainz, Germany. ${ }^{8}$ Genomic Medicine, Department of General, Transplant, and Liver Surgery, Medical University of Warsaw, Warsaw, Poland. 'Department of Pathology, Martin Luther University Halle-Wittenberg, Halle (Saale), Germany.

Received: 3 April 2013 Accepted: 30 September 2013 Published: 7 October 2013

\section{References}

1. Franc B, De-la SP, Lange F, Hoang C, Louvel A, De RA, Vilde F, Hejblum G, Chevret $\mathrm{S}$, Chastang C: Interobserver and intraobserver reproducibility in the histopathology of follicular thyroid carcinoma. Hum Pathol 2003, 34:1092-1100
2. Lang W, Georgii A, Stauch G, Kienzle E: The differentiation of atypical adenomas and encapsulated follicular carcinomas in the thyroid gland. Virchows Arch A Pathol Anat Histol 1980, 385:125-141.

3. Cheung L, Messina M, Gill A, Clarkson A, Learoyd D, Delbridge L, Wentworth J, Philips J, Clifton-Bligh R, Robinson BG: Detection of the PAX8-PPAR gamma fusion oncogene in both follicular thyroid carcinomas and adenomas. J Clin Endocrinol Metab 2003, 88:354-357.

4. Fagin JA, Mitsiades N: Molecular pathology of thyroid cancer: diagnostic and clinical implications. Best Pract Res Clin Endocrinol Metab 2008, 22:955-969.

5. Sahin M, Allard BL, Yates M, Powell JG, Wang XL, Hay ID, Zhao Y, Goellner JR, Sebo TJ, Grebe SK, et al: PPARgamma staining as a surrogate for PAX8/PPARgamma fusion oncogene expression in follicular neoplasms: clinicopathological correlation and histopathological diagnostic value. J Clin Endocrinol Metab 2005, 90:463-468.

6. Esapa CT, Johnson SJ, Kendall-Taylor P, Lennard TW, Harris PE: Prevalence of Ras mutations in thyroid neoplasia. Clin Endocrinol (Oxf) 1999, 50:529-535.

7. Shi YF, Zou MJ, Schmidt H, Juhasz F, Stensky V, Robb D, Farid NR: High rates of ras codon 61 mutation in thyroid tumors in an iodide-deficient area. Cancer Res 1991, 51:2690-2693.

8. Suarez HG, du Villard JA, Severino M, Caillou B, Schlumberger M, Tubiana M, Parmentier $C$, Monier R: Presence of mutations in all three ras genes in human thyroid tumors. Oncogene 1990, 5:565-570.

9. Vasko V, Ferrand M, Di CJ, Carayon P, Henry JF, De MC: Specific pattern of RAS oncogene mutations in follicular thyroid tumors. J Clin Endocrinol Metab 2003, 88:2745-2752.

10. Saggiorato E, De PR, Volante M, Cappia S, Arecco F, Dei Tos AP, Orlandi F, Papotti M: Characterization of thyroid 'follicular neoplasms' in fine-needle aspiration cytological specimens using a panel of immunohistochemical markers: a proposal for clinical application. Endocr Relat Cancer 2005 12:305-317.

11. Abulkheir IL, Mohammad DB: Value of immunohistochemical expression of p27 and galectin-3 in differentiation between follicular adenoma and follicular carcinoma. Appl Immunohistochem Mol Morphol 2012, 20:131-140.

12. Bryson PC, Shores CG, Hart C, Thorne L, Patel MR, Richey L, Farag A, Zanation AM: Immunohistochemical distinction of follicular thyroid adenomas and follicular carcinomas. Arch Otolaryngol Head Neck Surg 2008, 134:581-586

13. Cochand-Priollet B, Dahan H, Laloi-Michelin M, Polivka M, Saada M, Herman P, Guillausseau PJ, Hamzi L, Pote N, Sarfati E, et al: Immunocytochemistry with cytokeratin 19 and anti-human mesothelial cell antibody (HBME1) increases the diagnostic accuracy of thyroid fine-needle aspirations: preliminary report of 150 liquid-based fine-needle aspirations with histological control. Thyroid 2011, 21:1067-1073.

14. Tan A, Etit D, Bayol U, Altinel D, Tan S: Comparison of proliferating cell nuclear antigen, thyroid transcription factor-1, Ki-67, p63, p53 and high-molecular weight cytokeratin expressions in papillary thyroid carcinoma, follicular carcinoma, and follicular adenoma. Ann Diagn Pathol 2011, 15:108-116.

15. Paunovic I, Isic T, Havelka M, Tatic S, Cvejic D, Savin S: Combined immunohistochemistry for thyroid peroxidase, galectin-3, CK19 and HBME-1 in differential diagnosis of thyroid tumors. APMIS 2012, 120:368-379.

16. Nikiforova MN, Tseng GC, Steward D, Diorio D, Nikiforov YE: MicroRNA expression profiling of thyroid tumors: biological significance and diagnostic utility. J Clin Endocrinol Metab 2008, 93:1600-1608.

17. Weber F, Teresi RE, Broelsch CE, Frilling A, Eng C: A limited set of human MicroRNA is deregulated in follicular thyroid carcinoma. J Clin Endocrinol Metab 2006, 91:3584-3591.

18. Rossing M, Borup R, Henao R, Winther O, Vikesaa J, Niazi O, Godballe C, Krogdahl A, Glud M, Hjort-Sorensen C, et al: Down-regulation of microRNAs controlling tumourigenic factors in follicular thyroid carcinoma. J Mol Endocrinol 2012, 48:11-23.

19. Borup R, Rossing M, Henao R, Yamamoto Y, Krogdahl A, Godballe C, Winther O, Kiss K, Christensen L, Hogdall E, et al: Molecular signatures of thyroid follicular neoplasia. Endocr Relat Cancer 2010, 17:691-708.

20. Chevillard S, Ugolin N, Vielh P, Ory K, Levalois C, Elliott D, Clayman GL, El-Naggar AK: Gene expression profiling of differentiated thyroid neoplasms: diagnostic and clinical implications. Clin Cancer Res 2004, 10:6586-6597. 
21. Hinsch N, Frank M, Doring C, Vorlander C, Hansmann ML: QPRT: a potential marker for follicular thyroid carcinoma including minimal invasive variant; a gene expression, RNA and immunohistochemical study. BMC Cancer 2009, 9:93.

22. Lubitz CC, Gallagher LA, Finley DJ, Zhu B, Fahey TJ III: Molecular analysis of minimally invasive follicular carcinomas by gene profiling. Surgery 2005, 138:1042-1048.

23. Takano T, Miyauchi A, Yoshida H, Kuma K, Amino N: High-throughput differential screening of mRNAs by serial analysis of gene expression: decreased expression of trefoil factor 3 mRNA in thyroid follicular carcinomas. Br J Cancer 2004, 90:1600-1605.

24. Weber F, Shen L, Aldred MA, Morrison CD, Frilling A, Saji M, Schuppert F, Broelsch CE, Ringel MD, Eng C: Genetic classification of benign and malignant thyroid follicular neoplasia based on a three-gene combination. J Clin Endocrinol Metab 2005, 90:2512-2521.

25. Fryknas M, Wickenberg-Bolin U, Goransson H, Gustafsson MG, Foukakis T, Lee JJ, Landegren U, Hoog A, Larsson C, Grimelius L, et al: Molecular markers for discrimination of benign and malignant follicular thyroid tumors. Tumour Biol 2006, 27:211-220.

26. Cerutti JM, Delcelo R, Amadei MJ, Nakabashi C, Maciel RM, Peterson B, Shoemaker J, Riggins GJ: A preoperative diagnostic test that distinguishes benign from malignant thyroid carcinoma based on gene expression. J Clin Invest 2004, 113:1234-1242.

27. Chudova D, Wilde JI, Wang ET, Wang H, Rabbee N, Egidio CM, Reynolds J, Tom E, Pagan M, Rigl CT, et al: Molecular classification of thyroid nodules using high-dimensionality genomic data. J Clin Endocrinol Metab 2010, 95:5296-5304

28. Alexander EK, Kennedy GC, Baloch ZW, Cibas ES, Chudova D, Diggans J, Friedman L, Kloos RT, LiVolsi VA, Mandel SJ, et al: Preoperative diagnosis of benign thyroid nodules with indeterminate cytology. N Engl J Med 2012, 367:705-715.

29. Wu ZJ, Irizarry RA, Gentleman R, Martinez-Murillo F, Spencer F: A model-based background adjustment for oligonucleotide expression arrays. J Am Stat Assoc 2004, 99:909-917.

30. Slawski M, Daumer M, Boulesteix AL: CMA: a comprehensive Bioconductor package for supervised classification with high dimensional data. BMC Bioinformatics 2008, 9:439.

31. Vandesompele J, De PK, Pattyn F, Poppe B, Van RN, De PA, Speleman F: Accurate normalization of real-time quantitative RT-PCR data by geometric averaging of multiple internal control genes. Genome Biol 2002, 3:RESEARCH0034

32. Foukakis T, Gusnanto A, Au AY, Hoog A, Lui WO, Larsson C, Wallin G, Zedenius J: A PCR-based expression signature of malignancy in follicular thyroid tumors. Endocr Relat Cancer 2007, 14:381-391.

33. Saxen E, Franssila K, Bjarnason O, Normann T, Ringertz N: Observer variation in histologic classification of thyroid cancer. Acta Pathol Microbiol Scand A 1978, 86A:483-486.

34. Lange D, Sporny S, Sygut J, Kulig A, Jarzab M, Kula D, Jarzab B: [Histopathological diagnosis of thyroid cancer in a multicenter trial]. Endokrynol Pol 2006, 57:336-342.

35. Karger S, Krause K, Gutknecht M, Schierle K, Graf D, Steinert F, Dralle H, Fuhrer D: ADM3, TFF3 and LGALS3 are discriminative molecular markers in fine-needle aspiration biopsies of benign and malignant thyroid tumours. Br J Cancer 2012, 106:562-568.

36. England SK, Uebele VN, Kodali J, Bennett PB, Tamkun MM: A novel $\mathrm{K}+$ channel beta-subunit ( $\mathrm{hKv}$ beta 1.3) is produced via alternative mRNA splicing. J Biol Chem 1995, 270:28531-28534.

37. Lu R, Kanai N, Bao Y, Schuster VL: Cloning, in vitro expression, and tissue distribution of a human prostaglandin transporter cDNA(hPGT). J Clin Invest 1996, 98:1142-1149.

38. Gumienny TL, Brugnera E, Tosello-Trampont AC, Kinchen JM, Haney LB, Nishiwaki K, Walk SF, Nemergut ME, Macara IG, Francis R, et al: CED-12/ ELMO, a novel member of the Crkll/Dock180/Rac pathway, is required for phagocytosis and cell migration. Cell 2001, 107:27-41.

39. Hamm A, Veeck J, Bektas N, Wild PJ, Hartmann A, Heindrichs U, Kristiansen G, Werbowetski-Ogilvie T, Del MR, Knuechel R, et al: Frequent expression loss of Inter-alpha-trypsin inhibitor heavy chain (ITIH) genes in multiple human solid tumors: a systematic expression analysis. BMC Cancer 2008, 8:25.

40. Kinoshita M, Nakamura T, Ihara M, Haraguchi T, Hiraoka Y, Tashiro K, Noda M: Identification of human endomucin-1 and -2 as membrane-bound O-sialoglycoproteins with anti-adhesive activity. FEBS Lett 2001, 499:121-126.
41. Barden CB, Shister KW, Zhu B, Guiter G, Greenblatt DY, Zeiger MA, Fahey TJ III: Classification of follicular thyroid tumors by molecular signature: results of gene profiling. Clin Cancer Res 2003, 9:1792-1800.

42. Eszlinger M, Krohn K, Hauptmann S, Dralle H, Giordano TJ, Paschke R: Perspectives for improved and more accurate classification of thyroid epithelial tumors. J Clin Endocrinol Metab 2008, 93:3286-3294.

43. Yu S, Liu Y, Wang J, Guo Z, Zhang Q, Yu F, Zhang Y, Huang K, Li Y, Song E, et al: Circulating microRNA profiles as potential biomarkers for diagnosis of papillary thyroid carcinoma. J Clin Endocrinol Metab 2012, 97:2084-2092.

\section{doi:10.1186/1755-8794-6-38}

Cite this article as: Pfeifer et al:: Molecular differential diagnosis of follicular thyroid carcinoma and adenoma based on gene expression profiling by using formalin-fixed paraffin-embedded tissues. BMC Medical Genomics 2013 6:38.

\section{Submit your next manuscript to BioMed Central and take full advantage of:}

- Convenient online submission

- Thorough peer review

- No space constraints or color figure charges

- Immediate publication on acceptance

- Inclusion in PubMed, CAS, Scopus and Google Scholar

- Research which is freely available for redistribution

Submit your manuscript at www.biomedcentral.com/submit
C Biomed Central 